\title{
Adverse Events Associated With Synthetic Male Slings: An Analysis of the Food and Drug Administration Manufacturer and User Facility Device Experience Database
}

\author{
Hanson Zhao, Colby P. Souders, Paige K. Kuhlmann, Kai Dallas, Karyn Eilber, Jennifer T. Anger \\ Division of Urology, Department of Surgery, Cedars-Sinai Medical Center, Los Angeles, CA, USA
}

Purpose: We sought to describe and analyze the adverse events associated with synthetic male slings reported to the U.S. Food and Drug Administration (FDA) Manufacturer and User Facility Device Experience (MAUDE) database.

Methods: We queried the MAUDE database for all entries including the terms "Male Sling," "InVance," "Virtue," or "Advance" from January 1st, 2009 to December 31st, 2018. We collected and analyzed information about the event type, date received, report source, source type, and manufacturer. We reviewed and categorized the event description text for each medical device report (MDR).

Results: A total of 497 adverse events related to the male sling were identified. The adverse events were classified as injury (95.4\%), malfunction (4.2\%), and other (0.4\%). There were no deaths described. The slings involved were the Advance or Advance XP sling (69.8\%), InVance (15.5\%), Virtue Quadratic (12.3\%), or unknown (2.4\%). The 4 most common adverse events described were urinary incontinence (46.7\%), sling erosion (9.1\%), mechanical malfunction (8.2\%), and pain/numbness (8.2\%). There was no increase in the number of reports in the years following the FDA warnings for urogynecologic mesh.

Conclusions: There was an overall modest number of MDRs related to male slings and the majority of them were reported by the manufacturer. The reporting of adverse events for male slings does not seem to be affected by the controversy and scrutiny towards transvaginal mesh and midurethral slings. Further clinical studies and more objective and detailed databases are needed to investigate the safety of these synthetic slings.

Keywords: Male sling; Mesh; Male stress incontinence; Urethra; Erosion

- Conflict of Interest: JTA is an expert witness for Boston Scientific. KE is an expert witness and on the advisory board for Boston Scientific and a consultant for Coloplast.

\section{INTRODUCTION}

Male slings are used for the treatment of male stress urinary incontinence (SUI) [1,2]. Commonly used slings have included the InVance (American Medical Systems, Minnetonka, MN, USA), Advance/Advance XP (Boston Scientific, Marlborough, MA, USA), and the Virtue Quadratic (Coloplast, Humlebaek,
Denmark) [3-6]. These polypropylene or polyester synthetic mesh slings are implanted through a perineal approach and are designed to improve continence through urethral compression and relocation [1]. Although the male sling may not be as effective as an artificial urinary sphincter for men with severe SUI or a history of radiation, it continues to play an important role in the treatment algorithm for post-prostatectomy incontinence

Corresponding author: Jennifer T. Anger (DD https://orcid.org/0000-0002-7688-4688 Division of Urology, Department of Surgery, Cedars-Sinai Medical Center, $99 \mathrm{~N}$ La Cienega Blvd M102, Beverly Hills, CA 90211, USA

Email: Jennifer.Anger@cshs.org

Submitted: August 14, 2020 / Accepted after revision: October 20, 2020 
(PPI), and recent population-level studies have shown that volume of male slings has been steadily increasing [2,7-10].

While clinical trials and institutional series have described the outcomes of various male slings, the reported adverse events have been modest and may not be representative of the full spectrum of real-world complications [1]. The U.S. Food and Drug Administration (FDA) Manufacturer and User Facility Device Experience (MAUDE) database was created to record adverse events involving all medical devices [11]. This database was designed to monitor the safety of surgical technologies and is very familiar to urologists and urogynecologists as the controversy around transvaginal mesh (TVM) for pelvic organ prolapse (POP) stemmed from thousands of medical device reports (MDRs) in the MAUDE database [12].

In this study, we sought to describe and analyze the adverse events associated with male slings through the FDA MAUDE database. We hypothesize that the annual number of MDRs may be increasing due to the higher volume of male slings performed and the increased scrutiny towards synthetic mesh in urology.

\section{MATERIALS AND METHODS}

The study was determined to be exempt by our Institutional Review Board as this database is readily accessible to the public. The MAUDE database records adverse events associated with medical devices in the form of device malfunctions, injuries, and deaths. These adverse events are submitted by mandatory reporters (manufacturers, importers, and device user facilities) and voluntary reporters (healthcare professionals, patients, and consumers).

We queried the MAUDE database (https://www.accessdata. fda.gov/scripts/cdrh/cfdocs/cfMAUDE/search.CFM) for all entries with the terms "Male Sling," "InVance," "Virtue," or "Advance." As the web search is only limited to the last 10 years of data, we exported reports from January 1st, 2009 to December 31st, 2018. We collected and analyzed information about the event type, date received, report source, source type, and manufacturer. Although each MDR is already classified as an injury, malfunction, death, or other, we further characterized each report by reviewing the event description text was reviewed for each MDR. Based on the event description, each report was classified into 1 of 8 categories: incontinence, erosion, mechanical malfunction, pain/numbness, infection, retention, bladder/ urethral injury, or other/unknown. We also reviewed the out- comes of the adverse event if it was mentioned in the event description. Duplicate entries were removed as were entries that did not pertain to male slings.

\section{RESULTS}

A total of 497 adverse events related to the male sling were identified from January 1st, 2009 to December 31st, 2018. Fig. 1 shows the number of adverse events reported each year. The adverse events were classified on the MAUDE database as injury in 474 cases $(95.4 \%)$, malfunction in 21 cases $(4.2 \%)$, and other in 2 cases $(0.4 \%)$. There were no deaths reported. These adverse events were related to the Advance or Advance XP sling in 347 reports (69.8\%). The InVance sling was responsible for 77 reports (15.5\%), and the Virtue sling was responsible for 61 reports (12.3\%). A total of 12 reports (2.4\%) were due to a sling from American Medical Systems but the specific sling was unspecified.

Upon further review of the event description free text, we classified the adverse events into 8 categories. A total of 232 events $(46.7 \%)$ were related to urinary incontinence after sling placement. Sling erosion was described in 45 events (9.1\%). There was a mechanical malfunction when placing the sling for 41 events (8.2\%). A total of 41 events (8.2\%) were related to pain or numbness in the postoperative setting. The remainder were due to infection $(n=33)$, retention $(n=23)$, bladder/urethral injury $(n=15)$, or were unknown $(n=67)$. Fig. 2 shows the frequency of each event classification.

The report source was from a manufacturer for 490 reports (98.6\%). The rest were from a user facility (4 reports) or voluntary

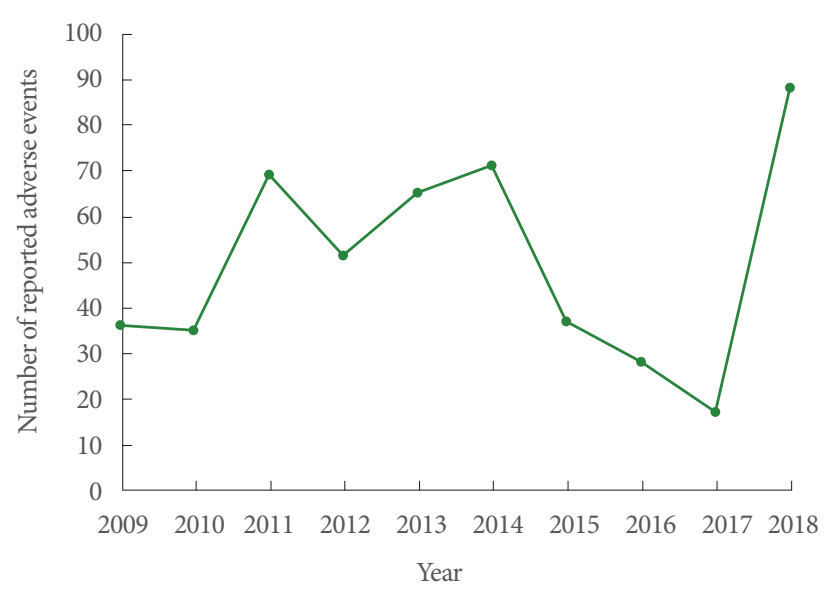

Fig. 1. Annual number of reported adverse events from 2009 to 2018. 


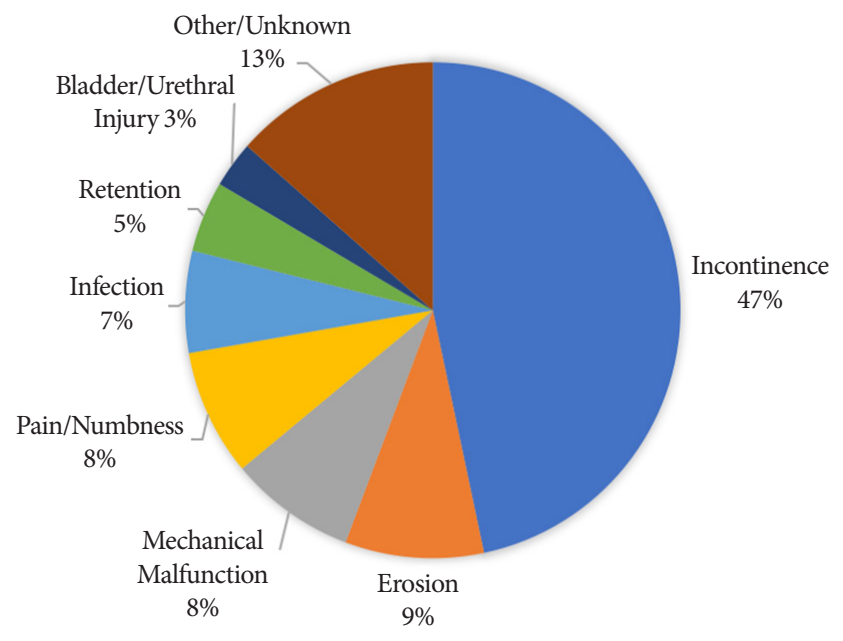

Fig. 2. Reasons for reported adverse events.

(3 reports). The source type was reported to be from a health care professional for 428 reports (86.1\%), consumer for 33 reports (6.6\%), or other for 36 reports ( $7.2 \%)$.

\section{DISCUSSION}

In this study, we identified 497 MDRs pertaining to male slings in the MAUDE database from 2009 to 2018. Most of the reports were about the Advance or Advance XP sling. There was an overall modest number of adverse events reported for male slings with an annual average of 49.7 reports. Almost all the reports in the study period were from the manufacturer and none were filed by an attorney. Many of the adverse events described were expected outcomes or known complications of the male sling.

The contrast between the MAUDE database on male slings and female midurethral slings (MUS) and TVM is notable. While the large number of MDRs in the MAUDE database started the controversy towards TVM in the late 2000s, the subsequent 2011 FDA safety communication on TVM resulted in a huge spike in the sheer volume of reports. Abraham found that the number of MDRs on TVM increased from 1,687 in 2011 to 11,710 in 2012 and 43,680 in 2013 [13]. A substantial number of these reports were filed by attorneys as well: 335 in 2011, 6,228 in 2012, and 8,219 in 2013 [13]. Although treatment for SUI was not included in the 2011 FDA safety communication or the 2016 FDA reclassification on TVM, these reports from the FDA have greatly impacted the usage and perception of MUS for SUI in females. The number of lawsuits filed for TVM increased from 730 in 2011 to 11,798 in 2012 to
34,017 in 2013 and the $63.3 \%$ of these involved MUS for SUI [14]. Ultimately, several mesh manufacturers have suffered significant financial consequences and have since removed their products from the market [15].

The number of MDRs on male slings actually decreased after the 2011 FDA safety communication from 69 in 2011 to 59 in 2012. In addition, none of the reports were filed by an attorney. These trends suggest that the scrutiny towards TVM has not yet had an identifiable effect on the reporting of male slings in the MAUDE database in the time period studied. The discrepancy in the sheer number of MAUDE reports could also be related to differences in the volume of slings performed. An analysis of Medicare data estimated that 33,880 slings were placed in 2007 for females and the FDA 2011 safety report estimated that over 200,000 women underwent a surgical repair of SUI with mesh in 2010 [16,17]. On the other hand, MacDonald et al. [18] analyzed the National (Nationwide) Inpatient Sample (NIS) database to find that only 11,635 sling procedures were performed for men over a 12-year period from 2000 to 2012. The number of male slings is likely underestimated as the NIS database only includes inpatient admissions. Nevertheless, based on this data, the volume of female slings is roughly several hundred times more than that of male slings. Interestingly, the number of MAUDE reports actually appears to correlate with that. There were 11,710 and 43,680 reports for TVM in 2012 and 2013 and only 51 and 65 reports for male slings in those 2 years, which is equivalent to a 230 and 672 -fold difference.

The limitations of the MAUDE reporting system are well established, and the volume of reports does not necessary correlate to the content of the reports [12]. Of all the 57,908 reports filed on transvaginal slings and mesh, $46 \%$ did not have an identifiable reporter and $26 \%$ were reported by a lawyer [13]. Analysis of these MDRs found that they rarely contain the catalog, model, and lot number of the mesh in question. In addition, Caron et al. [19] found that nearly 20\% of these reports have been revised. Due to the voluntary reporting nature, the validity and reliability of the MAUDE database for TVM have often been called into question. Even the home page of the MAUDE website claims: “... This passive surveillance system has limitations, including the potential submission of incomplete, inaccurate, untimely, unverified, or biased data. In addition, the incidence or prevalence of an event cannot be determined from this reporting system alone due to underreporting of events, inaccuracies in reports, lack of verification that the device caused the reported event, and lack of information about 
frequency of device use." [11]. Nevertheless, there clearly is a role for reporting systems like the MAUDE database, particularly for early identification of medical device malfunctions and injuries.

In our analysis of male sling MDRs, the report source was a manufacturer for nearly all of the reports. Manufacturers are mandatory reporters that must submit reports when they become aware that their devices have caused death or injury or have malfunctioned in a way such that a recurrence of the malfunction in a similar device could contribute to a death or serious injury. Thus, their reports should have more credibility. Many of the manufacturer reports on male slings described well-known side effects including persistent urinary incontinence (47\%), postoperative pain or numbness (8\%), and urinary retention (5\%). The remainder of the MDRs were more severe complications including: erosions (9\%), mechanical malfunctions (8\%), infection (7\%), and bladder or urethral injuries (3\%).

There could be several explanations why the massive media and litigious backlash against TVM and MUS for females has not had a noticeable effect on the MAUDE reporting of male slings. Differences in surgeon experience, surgical techniques, and indications may lead to variations in the perception and treatment of their complications. The male sling is implanted deep within the perineum and is covered by multiple layers of subcutaneous tissue, preventing any cutaneous extrusion. The female sling is only covered by a thin layer of vaginal epithelium and thus could be more easily palpable and prone to extrusion. Male slings are placed for PPI and can be seen as salvage operations related to their initial cancer treatment so patients may be more accepting of those complications. Ultimately, there may be differences in the psychosocial characteristics of the 2 patient populations and how they are targeted by the media [20].

Of note, there was a 5 -fold increase in the number of male sling MDRs from 17 in 2017 to 88 in 2018. As 2018 was the last year in this study, we cannot assess whether this increase represents an outlier or the start of a new trend. Upon further analysis of the MDRs in 2018, 75 of those reports (85\%) described worsening urinary incontinence that was treated with another device, either a new sling or an artificial urinary sphincter. Many of these descriptions were vague and lacked details. And although these reports were ultimately submitted to the MAUDE database by the manufacturer, these entries or reports may have been duplicated. As overreporting was a large critique of the MAUDE database for female TVM, it will be important to closely monitor these trends over the next few years. We are reminded of a similar controversy 3 decades ago where the media attention towards the unfounded risk of connective tissue disease from silicone breast implants ultimately led to the discontinuation of silicon testicular implants [21,22].

The limitations of our study are largely based on the limitations of the MAUDE database as a passive surveillance system with potentially inaccurate and unverified data. The MAUDE dataset does not represent the entire spectrum of complications related to the male sling in this time period studied. Not all complications were reported to this system and not all reports are considered complications. We were also unable to obtain the overall volume of slings placed in this time frame. In addition, our study did not include every brand of male sling including the adjustable models. However, whereas others have used algorithms to mine through the MAUDE database, we were able to manually review the free text to categorize each MDR as there were a relatively small number of MDRs on male slings. Nevertheless, we were limited by the actual content of the descriptive text. Ultimately, further clinical studies and more objective and detailed databases are needed to investigate the safety of these synthetic slings.

In conclusion, our study finds that there is a modest number of adverse events related to the male sling on the MAUDE database. The reporters are usually manufacturers and many of the reports describe well-known sequela including persistent incontinence and pain. The reporting of adverse events for male slings does not seem to be affected by the controversy and scrutiny towards TVM and MUS. Further clinical studies and more objective and detailed databases are needed to investigate the safety of these synthetic slings.

\section{ORCID}

$\begin{array}{ll}\text { Hanson Zhao } & 0000-0002-9660-3476 \\ \text { Colby P. Souders } & 0000-0002-3700-4844 \\ \text { Paige K. Kuhlmann } & 0000-0001-9547-6797 \\ \text { Kai Dallas } & 0000-0003-1817-1353 \\ \text { Karyn Eilber } & 0000-0002-4014-5007 \\ \text { Jennifer T. Anger } & 0000-0002-7688-4688\end{array}$

\section{AUTHOR CONTRIBUTION STATEMENT}

- Conceptualization: $H Z, C S, K D, K E, J A$

- Data curation: $H Z, C S$ 
- Formal analysis: $H Z, P K, J A$

- Methodology: $H Z, P K, K D, J A$

- Project administration: $J A$

- Writing-original draft: $H Z$

-Writing-review \& editing: $H Z, C S, P K, K D, K E, J A$

\section{REFERENCES}

1. Meisterhofer K, Herzog S, Strini KA, Sebastianelli L, Bauer R, Dalpiaz O. Male slings for postprostatectomy incontinence: a systematic review and meta-analysis. Eur Urol Focus 2020;6:575-92.

2. Kretschmer A, Nitti V. Surgical treatment of male postprostatectomy incontinence: current concepts. Eur Urol Focus 2017;3:364-76.

3. Fassi-Fehri H, Badet L, Cherass A, Murat FJ, Colombel M, Martin $\mathrm{X}$, et al. Efficacy of the InVance male sling in men with stress urinary incontinence. Eur Urol 2007;51:498-503.

4. Bauer RM, Grabbert MT, Klehr B, Gebhartl P, Gozzi C, Homberg R, et al. 36-month data for the AdVance $\mathrm{XP}^{\circledR}$ male sling: results of a prospective multicentre study. BJU Int 2017;119:626-30.

5. Leruth J, Waltregny D, de Leval J. The inside-out transobturator male sling for the surgical treatment of stress urinary incontinence after radical prostatectomy: midterm results of a single-center prospective study. Eur Urol 2012;61:608-15.

6. Comiter CV, Rhee EY, Tu LM, Herschorn S, Nitti VW. The virtue sling--a new quadratic sling for postprostatectomy incontinence-results of a multinational clinical trial. Urology 2014;84:433-8.

7. Chughtai B, Sedrakyan A, Isaacs AJ, Mao J, Lee R, Te A, et al. National study of utilization of male incontinence procedures. Neurourol Urodyn 2016;35:74-80.

8. Liu JS, Hofer MD, Milose J, Oberlin DT, Flury SC, Morey AF, et al. Male sling and artificial urethral sphincter for male stress urinary incontinence among certifying American Urologists. Urology 2016; 87:95-9.

9. Comiter CV, Dobberfuhl AD. The artificial urinary sphincter and male sling for postprostatectomy incontinence: which patient should get which procedure? Investig Clin Urol 2016;57:3-13.

10. Averbeck MA, Woodhouse C, Comiter C, Bruschini H, Hanus T, Herschorn S, et al. Surgical treatment of post-prostatectomy stress urinary incontinence in adult men: report from the 6th International Consultation on Incontinence. Neurourol Urodyn 2019;38: 398-406.

11. U.S. Food and Drug Administration. MAUDE - Manufacturer and user facility device experience [Internet]. Silver Spring (MD): U.S. Food and Drug Administration; 2021 [cited 2019 Aug 1]. Available from: https://fda.report/MAUDE/.

12. Sandberg JM, Gray I, Pearlman A, Terlecki RP. An evaluation of the manufacturer and user facility device experience database that inspired the United States Food and Drug Administration's reclassification of transvaginal mesh. Investig Clin Urol 2018;59:126-32.

13. Abraham A, Tzartzeva K, Christie A, Zimmern P. Lessons learned from the MAUDE database on transvaginal synthetic mesh and sling reports. Urol Pract 2017;4:111-7.

14. Souders CP, Eilber KS, McClelland L, Wood LN, Souders AR, Steiner V, et al. The truth behind transvaginal mesh litigation: devices, timelines, and provider characteristics. Female Pelvic Med Reconstr Surg 2018;24:21-5.

15. Cohn J, Brown E, Kowalik C, Kaufman M, Dmochowski R, Reynolds WS. The mesh controversy [version 1; peer review: 2 approved]. F1000Research 2016;5(F1000 Faculty Rev):2423.

16. Rogo-Gupta L, Litwin MS, Saigal CS, Anger JT, Project UDiA. Trends in the surgical management of stress urinary incontinence among female Medicare beneficiaries, 2002-2007. Urology 2013;82: 38-41.

17. U.S. Food and Drug Administration. Urogynecologic surgical mesh: update on the safety and effectiveness of transvaginal placement for pelvic organ prolapse [Internet]. Silver Spring (MD): U.S. Food and Drug Administration; 2021 July [2019 Aug 1]. Available from: https://www.fda.gov/media/81123/download.

18. MacDonald S, Colaco M, Terlecki R. Waves of change: national trends in surgical management of male stress incontinence. Urology 2017;108:175-9.

19. Caron A, Colby S, Obi N, Clark K, Eilber K, Anger JT. Efficacy of self-reported databases. In: Society for Urodynamics, Female Pelvic Medicine \& Urogenital Reconstruction 2019 Winter Meeting; 2019 Feb 26-Mar 2; Miami, Florida; Linthicum (MD): American Urological Association; 2019.

20. Rosenbaum L. N-of-1 policymaking--tragedy, trade-offs, and the demise of morcellation. N Engl J Med 2016;374:986-90.

21. Angell M. Shattuck Lecture--evaluating the health risks of breast implants: the interplay of medical science, the law, and public opinion. N Engl J Med 1996;334:1513-8.

22. Randall T. Penile, testicular, other silicone implants soon will undergo FDA review. JAMA 1992;267:2578-9. 